
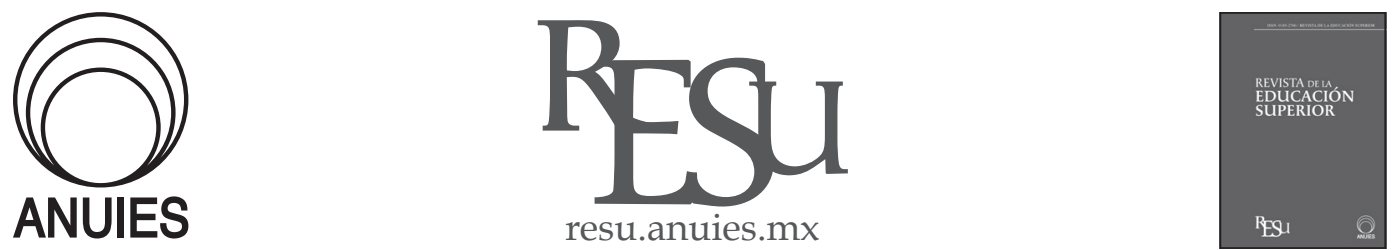

Revista de la Educación Superior 48 (190) (2019)

ARTÍ́CULO

\title{
La universidad en crisis, ¿amenaza o reafirmación de su ontología?
}

\section{The university in crisis, does it threaten or reaffirm its ontology?}

\section{Germán Alonso Vélez Sánchez* y Gustavo Alberto Ruiz Rojas}

Fuente de financiación: El artículo es un ensayo académico resultado de la investigación: Trayectos de la investigación en la Universidad Autónoma Latinoamericana a través de la deconstrucción de sus metáforas dominantes. Esta se desarrolla en el marco de la convocatoria CONVDI2016-01 de la dirección de investigaciones de dicha universidad ubicada en la ciudad de Medellín, Colombia.

Agradecimientos: A la Universidad Autónoma Latinoamericana por su apoyo financiero para el desarrollo de la investigación que dio como uno de sus resultados este original tipo ensayo.

* Universidad Autónoma Latinoamericana. Medellín, Colombia. Correo electrónico: german.velezsa@unaula.edu.co

Recibido el 22 de marzo del 2018; aceptado el 25 de febrero del 2019.

\section{Resumen}

El artículo aborda la cuestión sobre la idea de universidad. Mediante un diseño cualitativo de corte hermenéutico, se interpretaron las ideas dominantes acerca de la razón de ser y la misión de la Universidad. Se develaron tensiones históricas entre las lógicas técnico-instrumentales de corte productivo y la tendencia crítica que aboga por una universidad para la humanidad, centrada en el compromiso con la sociedad, la cultura y el planeta. Se analizan los principales referentes que han cimentado las bases teóricas para una concepción de la universidad alternativa que atiende a la diversidad ancestral y cultural.

Palabras clave: Universidad; Educación; Ontología; Investigación; Crisis. 


\begin{abstract}
The article addresses the issue of the idea of university. Through a qualitative design of hermeneutic approach, dominant ideas about the raison d'être or reason to be and mission of the University were interpreted. Historical tensions were revealed between the technical-instrumental logics of productive scheme and the critical tendency that advocates a university for humanity, focused on the commitment to society, culture and the planet. We analyze the main references that have laid the theoretical foundations for a conception of the alternative university that serves ancestral and cultural diversity.
\end{abstract}

Keywords: University; Education; Ontology; Research; Crisis.

\title{
Introducción
}

$\mathrm{E}$ n "De la idea de Universidad a la Universidad de las ideas", Santos, (1998) menciona la permanencia histórica de los fines de la universidad: la búsqueda sistemática de la verdad y su transmisión que más allá de la propia ciencia involucra la cultura. Sustenta que a partir de la década de 1960 esta tradición le asigna a la universidad tres objetivos: investigación, enseñanza y extensión. Con ello se atrofia su dimensión cultural y se genera una visión productivista que deriva en múltiples funciones, algunas de ellas contradictorias entre sí, conducentes a diversas tensiones que han puesto en cuestión la legitimidad de la universidad.

La primera tensión se da entre formación de alto nivel orientada a las élites y la de formación especializada enfocada a la productividad (Crisis de hegemonía), lo que deriva en la búsqueda de otras opciones para satisfacer las demandas de formación; la segunda, entre la jerarquización de los saberes, emanados de la especialización y el conocimiento y las exigencias de democratización e igualdad de oportunidades (Crisis de legitimidad), con la consecuente falta de consenso acerca del valor de la universidad y de su importancia en el contexto social; la tercera, entre las aspiraciones de autonomía y la sumisión a los criterios de eficacia y de productividad de origen empresarial (Crisis institucional), lo que origina una crítica a la pertinencia de los procesos propios de las universidades y la exigencia de adoptar otros emanados del sector productivo, desconociendo así que la pertinencia es un concepto relacional que se articula a partir de los vínculos entre la universidad y distintos actores, lo que exige superar una concepción economicista del concepto para buscar visiones integrales que incorporen una mirada social y política del mismo (Malagón Plata, 2003) 
Este artículo tiene como propósito comprender la crisis de la universidad como una de las principales consecuencias de la implementación de los modelos hegemónicos que se han instalado, a partir de la aplicación de discursos de control, parametrización, rendimiento y productividad.

En primer lugar, se presentan los antecedentes de investigación; allí se realiza un recorrido por los tres grandes escenarios por los que ha transitado la producción investigativa acerca de la universidad a partir del denominado pacto de Bolonia y sus consecuencias. En segundo lugar se integran discusiones teóricas de índoles sociológica y filosófica que debaten el sentido de la universidad y critican a la universidad en el contexto del mercado. Por último, se genera una discusión acerca de la universidad para América Latina en una perspectiva decolonial.

\section{Antecedentes para una universidad en crisis ontológica}

La universidad debe discutir permanentemente el sentido de su razón de ser, especialmente ante las demandas sociales que entran en contradicción con lo planteado en los estatutos de la Universidad Moderna (Llamas Tascón, 1997). Por ello, diversas producciones académicas han reiterado la necesidad de repensar el papel de la universidad.

Se recalca que las nuevas exigencias sociales tocan aspectos metodológicos, formativos, de gestión y de transformación a partir de las tecnologías de la información y la comunicación (Lampert, 2009; Mateo \& Vlachopoulos, 2013). Desde este punto de vista, "La universidad está siendo interpelada por preguntas complejas para las que hasta el momento sólo ha producido respuestas simples" (Santos B., 2010).

Algunos sujetan las concepciones de la nueva universidad a la idea de postmodernidad. A pesar del debate acerca de la validez sociológica del concepto, se indica que la universidad actual está lejos del ideal de la modernidad, por la necesidad de masificar su alcance, la desfinanciación, la pérdida de valor de las titulaciones, la gestión de corte empresarial y la influencia de las nuevas tecnologías de la información (García Ruiz, 2011).

Se afirma que la universidad debe cambiar sustancialmente, porque el conocimiento ya no es universal, ni es privilegio de una élite académica. Así, la misión de la universidad es preparar individuos para responder a una cultura laboral crecientemente diversificada (García Ruiz, 2011). Esto implica el desarrollo de un conocimiento tipo 2, de carácter no jerárquico, flexible y adaptado a las 
necesidades de los estudiantes y las empresas, lo cual incrementa las investigaciones aplicadas orientadas a captar recursos (García Ruiz, 2011).

Entonces, la universidad debería emprender una nueva misión. Se trata de la "tercera misión", sustentada en aplicación del conocimiento, innovación, formación laboral cualificada, fomento a la investigación y el desarrollo de proyectos emprendedores (ACUP, 2008, citado por García Peñalvo, 2016). Esta misión permitirá reducir la atávica distancia entre empresa y universidad mediante la triple hélice empresa-universidad-administración: “[...] Las instituciones terciarias, no universitarias, de orientación predominantemente profesional o técnico profesional, alcanzan en este nuevo ámbito un rango equivalente al universitario" (Alonso Bra, 2008: 81).

Se recalca que la globalización, las nuevas tecnologías, la emigración, la movilidad y la formación continua exigen una universidad más real que funcione como empresa de cara al mercado, por lo cual se requiere de un profesorado más profesional (Olivera Beltrán, 2000).

Los anuncios de transformación hacia una sociedad del conocimiento aparecen discursivamente como una realidad totalizante ante la cual la universidad debe responder, para ello el Espacio Común Europeo emerge como salida (Montero Curiel, 2010). El pacto de Bolonia enfatiza el carácter internacional de la educación para garantizar la comparabilidad y la movilidad de los estudiantes, futuros trabajadores, en los contextos de integración entre Estados, lo que le permite a Europa fomentar su crecimiento económico, su competitividad internacional y su cohesión social (Bombillar Sánez, 2010; Egido Álvarez, Fernández Díaz, \& Galán, 2014).

Esto implica la formación multilingüe y por competencias, la orientación al trabajo, y el énfasis en metodologías de enseñanza centradas en el estudiante y su posibilidad de generar nuevo conocimiento (Gijón Puerta \& Crisol Moya, 2012). Lo cual exige el cambio de una enseñanza centrada en el esfuerzo del profesor, hacia una centrada en el esfuerzo del estudiante, con el fin de lograr desde la formación una mayor integración social y generación de empleo (Calvo Bernardino \& Mingorance Arnaiz, 2009).

Todo lo anterior reconfigura la esencia y la misión del profesor quien resulta vital para lograr una educación centrada en el desarrollo de competencias para el trabajo como el punto nodal del acuerdo de Bolonia (Mas-Torelló \& OlmosRueda, 2016), necesarias en virtud de la brecha entre oferta, conocimientos teóricos académicos, y demanda, conocimientos prácticos requeridos por la empresa (Bartual Figueras \& Turmo Garuz, 2016).

Por tal razón, los profesores deben cambiar la orientación de las tutorías de un enfoque meramente académico a una mirada más profesionalizante y un 
acompañamiento personalizado (García Nieto, Asensio Muñoz, Carballo Santaolalla, García García, \& Guardia González, 2005), además de las metodologías para que el docente garantice el aprendizaje activo y autorregulado del estudiante (Ion \& Cano, 2012), y también el fortalecimiento de actitudes democráticas de los "usuarios" de la educación mediante un enfoque menos centrado en las metas (Huber, 2008), lo cual demanda una evaluación sistemática de las prácticas pedagógicas de los docentes, en virtud del cambio de roles entre profesor y estudiante. Esto significa que el profesor “[...] debe actuar como mediador, como diseñador de entornos de aprendizaje y como propiciador del aprendizaje autónomo de los alumnos" (Gargallo, Suárez, Garfella, \& Fernández, 2011: 10).

Un asunto sensible radica en la formación de competencias docentes para el aprovechamiento de las nuevas tecnologías de la información que garanticen versatilidad y flexibilidad en el proceso de enseñanza (Alba Pastor \& Carballo Santaolalla, 2005; De Pons \& Villaciervos Moreno, 2005).

El propósito es lograr un ciudadano concebido como recurso y como capital para participar en la sociedad del conocimiento, lo que implica que tenga derecho a acceder a mecanismos para la transformación de la información en conocimiento, por lo cual el e-learning se convierte en un medio de acceso privilegiado garante del acceso equitativo a la educación (Trujillo, Hinojo Lucena, \& Aznar Díaz, 2011). Por lo tanto, se requiere que los docentes vayan más allá de "un posicionamiento crítico sin más" en beneficio de una actitud desde el trabajo de campo comprometido, expresado en nuevas formas de transmitir la información para convertirla en conocimiento, en la motivación para participar dentro de procesos de desarrollo, y el énfasis en los procesos, así como en el diseño de ambientes multimodales para el aprendizaje (Trujillo, Hinojo Lucena, \& Aznar Díaz, 2011).

El Espacio Europeo de Educación Superior EeEs definió la estructura de la educación superior desde dos ciclos: grado y posgrado. El doctorado aparece como una ampliación del posgrado. Así, la formación para la investigación que se produce en el doctorado fue considerada como una extensión del posgrado, perdiendo su identidad respecto de la alta responsabilidad de formar investigadores. Para corregir este vacío, la Declaración de Berlín de 2003 lo incluye como tercer ciclo de formación que completa los definidos por la Declaración de Bolonia, con el fin de construir las bases de la Sociedad del Conocimiento (Jiménez-Ramírez, 2017) .

Exceptuando a Ibarra Colado (2002), los trabajos anteriormente reseñados tienen una unidad discursiva articulada como un régimen de verdad y agenciada sobre la base del sentido común que, al ser incorporado en el lenguaje, afecta el sentido de identidad, inhibe la reflexión sobre la acción y crea unos principios 
indiscutibles que deben replicarse (Torres, 2014: 21). Tal es el caso de algunas críticas que apuntan más a cuestionar la forma de implementar el acuerdo que al acuerdo mismo. Se destaca la discusión acerca del papel del profesor universitario, el cual no se menciona como agente activo dentro de los acuerdos (Alba Pastor, 2005), por lo que los docentes reclaman que se les haya impuesto un cambio sin contar con la información suficiente para entender las dimensiones e implicaciones del mismo, como también exigen procesos de formación para tal efecto (Cabello Martínez \& Antón Ares, 2005; Margaleff García \& Álvarez Méndez, 2005; Michavila, 2005).

Una actitud crítica frente a las posturas afirmativas y naturalizantes del acuerdo de Bolonia denuncia el pensamiento único subyacente en sus desarrollos investigativos (Cuño Bonito, 2016). Santos (2010) afirma que transcurrida una década desde el inicio del proceso de Bolonia, sólo se han presentado silencios y naturalizaciones que imponen un nuevo sentido común acerca de la misión de la universidad, con el peligro de que logros mediocres se interpreten como brillantes saltos adelante y que se caiga, con la máscara del consenso, en una resignación que amenaza el sentido de la universidad. Por tal razón, algunas visiones críticas han insistido en la necesidad de restituir su sentido ontológico.

En primer lugar, se encuentra el retorno a la idea de Universidad anclada en la formación humboltiana de corte alemán. Ésta reclama una formación humanística y una relación reflexiva con la ciencia (Steger, citado por Benítez, 1996). Kurt L. Shell insiste en la necesidad de la formación humanística pero orientada a fortalecer la democracia (citado por Benítez, 1996). Sin embargo, es evidente la reducción de cursos humanísticos dentro de la formación universitaria a partir del pacto de Bolonia, en especial los estudios literarios, demostrando la vocación pragmática del acuerdo y el desconocimiento del valor formativo de la literatura en la construcción de ciudadanía, con lo cual las declaraciones de formación ciudadana emanadas del pacto de Bolonia resultan insustanciales (Ibarra \& Ballester, 2016).

Burton Clark anticipó que el crecimiento en la matrícula de estudiantes universitarios generaría una presión sobre los procesos de investigación de las universidades, lo cual derivó en una segmentación en diferentes niveles: una profesionalizante en pregrado la cual pretende satisfacer las empresas ancladas aún en un enfoque Taylorista, y una investigativa de élite en posgrado, con fin de capturar a los mejores investigadores que contribuye a la generación de conocimiento necesaria para participar del capitalismo académico (Rodríguez Gómez, 2001,Ibarra Colado, 2002). Así, el habitus del docente universitario se vincula a una investigación cada vez más científico-técnica y económico-funcional del 
mundo, lo que deriva en una reproducción simbólica del esquema tecno-científico (Runge Peña, 2005).

Como consecuencia, se produce la transformación del conocimiento en mercancía, la proletarización ideológica y económica del profesor universitario, el culto a la información como base para el desarrollo con lo cual se llega a la desmaterialización de las relaciones económicas, las universidades como eco de las empresas y su orientación hacia la formación de sujetos flexibles y adaptables, en otras palabras, dóciles a los discursos psicologistas y gerencialistas que intentan fomentar la adaptabilidad (Vega Cantor, 2015).

Entonces, la universidad se ha movido entre la búsqueda de la verdad que contribuye al espíritu científico y la formación humanística que fortalece la formación del sujeto. En ese sentido, remarca la misión del docente de formar en la verdad y no en el éxito y la realización individual como propósito de la universidad actual, en la cual el concepto de verdad se diluye y se reemplaza por términos como "empleabilidad, competencia, adaptabilidad, internacionalismo, eficacia, etc." (Llano, 2011, citado por Ahedo Ruiz, 2016: 518), por lo cual la universidad tiene la necesidad de reconstruir su esencia fundacional sobre la base de la "pedagogización" de los problemas de la universidad y su politización no partidista, para con ello contribuir a la conquista de un cambio social emancipatorio (Mollis, 2014).

Según Guillermo Hoyos (2008), estas críticas más que rechazar el pensamiento científico, reivindican un nuevo humanismo que permite enfrentar las naturalizaciones y crisis de valores propias del desarrollo tecnocientífico. Un humanismo que reconoce los avances y poder formativo del pensamiento científico, pero que legitime las artes y el pensamiento filosófico como aspectos claves en la construcción de ciudadanía planetaria, para contribuir a la formación ciudadana y al desarrollo de una política deliberativa.

La segunda tendencia crítica piensa el sentido de la universidad en el contexto de América Latina. Desde una perspectiva sociológica, la universidad latinoamericana se enfrenta a varios retos como el crecimiento demográfico frente a la cobertura, la pertinencia y la contribución a la equidad mediante una mejor distribución del ingreso, garantizada a través de un mayor acceso a la educación superior (Rodríguez Gómez, 2001). Algunos enfoques como el de Rodríguez tienen una mirada social, en cambio, otras perspectivas pueden descansar en un enfoque liberal-progresista, que no está exento de servir como herramienta de dominación hegemónica y por lo tanto pueden ser una derivación de postulados desarrollistas (Torres, 2014).

Estos últimos le exigen a la universidad que se adapte a una nueva realidad mundial con eficiencia; afirman que la universidad debe cambiar o extinguirse 
por su obsolescencia y que debe "reformular la didáctica, los objetivos de enseñanza, el curriculum, la investigación y el financiamiento" (Geneyro, 1997: 247); sustentan que América Latina no es ajena a los nuevos retos propios de la sociedad del conocimiento; en consonancia con los propósitos de la Organización para la Cooperación y el Desarrollo Económicos (OCDE), plantean las circunstancias que justifican una nueva visión para la universidad, no pensada sólo como impulsora de sabiduría, sino también con una función de desarrollo económico, fundamento de mejores condiciones de desarrollo social e igualdad de oportunidades (Salcines, Freire Seoane, \& Orosa González, 2008).

Por tal razón, la ubicuidad del sentido investigativo de la universidad ha conducido a que muchas entidades de educación superior en Latinoamérica hayan empleado grandes esfuerzos y recursos, sin contar con las características estructurales para hacerlo, descuidando otras funciones de la universidad (Canales, 2011).

Pero también existen propuestas alternativas que promueven el rol de las universidades al servicio del bien común y postulan la necesidad de sostenerlas gracias al financiamiento público (Santos B. D, 1998; Rodríguez Gómez, 2001;Torres, 2014). La financiación pasa a ser factor decisivo en tanto las nuevas exigencias de calidad han repercutido sobre sus procesos de gestión, pues la generación de recursos depende de la satisfacción de esas exigencias (De Vries \& Ibarra Colado, 2004).

En el caso de la universidad pública, se afirma que ésta, por su naturaleza, no está orientada al lucro, por lo cual la evaluación de la gestión debe hacerse con base en la claridad sobre su misión y su impacto que, en América Latina, constituyen la formación de seres humanos orientados al bien común, lejos de posturas competitivas y mercantilistas (Lozada Barahona \& Gutiérrez Vargas, 2014).

Las discusiones evidencian el contraste entre una universidad al servicio de los postulados desarrollistas, con énfasis en la planeación, la hiper-especialización, el cientificismo social y la formación de recurso humano (Vasconi y Reca, citados por Torres, 1997), y una universidad que responda a los ideales de democracia, de gratuidad y de autonomía popular propuestos en el Manifiesto de Córdoba de 1918 (Torres, 1997); una universidad que encarne los principios democráticos del poder popular, que abra las aulas universitarias a las realidades de la comunidad, que sea sensible a sus valores y que reconozca sus saberes y prácticas como base de un diálogo simétrico (Castro Estrada, Rodríguez Rejas, \& Urteaga Urías, 2016). Se concluye a partir de estos argumentos, que la empresarialización de la universidad se afianza mediante la difusión de ideas que contribuyeron desde diferentes escenarios a desprestigiar la educación reformista en algunas universidades de América Latina, para dar paso así a la 
reducción del papel del Estado en la educación superior, lo cual, en nombre de la racionalidad del gasto, contribuyó a cambiar la idea del buen ciudadano por la del sujeto económico y consumidor (Mollis, 2014). Así, la idea moderna de Universidad, centrada en ideales democráticos, deviene en una realidad fundada en la enajenación de las cosas, las personas y las conciencias, para garantizar la acumulación individual de ganancias a partir del ejercicio de la racionalización". (Ibarra Colado, 2005: 24)

Ante la hegemonía de las presiones externas, la universidad debe reaccionar encontrando en su vitalidad endógena los horizontes que constituyen su esencia y su fuerza para oponerse, esgrimiendo su autonomía como bandera (Cuño Bonito, 2016). Para ello cuenta con estrategias que involucran una política pública de financiación, el fortalecimiento de la democracia universitaria, el desarrollo de procesos de control y gestión autónomos, las auditorías a la calidad universitaria cimentadas en una política pública de educación superior y el fortalecimiento de la universidad intercultural pensada en función de solucionar los problemas de las comunidades mediante un diálogo de saberes (Santos B. D., 1998; Cuño Bonito, 2016).

La visión intercultural de la educación superior demanda, desde la perspectiva latinoamericana, una conceptualización clara que supere las visiones europeas centradas en la asimilación cultural exigida para procesos de movilidad e integración (Muñoz Sedano, 1998). La Universidad Intercultural surge como respuesta a la carencia de cobertura universitaria entre las comunidades indígenas (Schmelkes, S. F.) y a la ausencia de propuestas adecuadas a los valores, necesidades y cosmovisión propios de estas comunidades; es una deuda histórica que demanda de la Universidad una redefinición de su esencia para "[...] ofrecer una formación que combine excelencia académica con pertinencia cultural y lingüística" (Mateos Cortés \& Dietz, 2016: 684). Se orienta a partir de una política reivindicatoria de los derechos de los pueblos indígenas en relación con el Estado y con la sociedad global, lo que en cada país de América Latina ha tomado diversas concepciones dependiendo de las dinámicas de fortalecimiento político e institucionalización de las comunidades indígenas, como el caso de Ecuador y Bolivia, o de las características propias de exclusión, como en el caso de Brasil (Williamson, 2004).

De allí que sea necesario precisar su sentido, al enunciarla como una educación que responda a las características y necesidades de los contextos en los cuales se dé y se oriente el reconocimiento de las formas particulares de entender el mundo (Castillo Rosas, 2016). Para ello se necesita la formación de jóvenes indígenas desde un diálogo de saberes, anclado en "la ecología de saberes" propuesta por Boaventura de Souza Santos (2007), para trascender el simple intercambio 
epistémico y la indistinción entre saberes teóricos y saberes prácticos. Este intercambio parte del reconocimiento de los diversos impulsos utópicos, lo que no permite asumir como central o hegemónica cualquier postura (Ávila Romero, Betancourt Posada, Arias Hernández, \& Ávila Romero, 2016). Estos saberes han de internalizarse como saberes prácticos que trasciendan conocimientos abstractos y descontextualizados y permitan una interacción dialógica entre el poder, el saber y el hacer (Mateos Cortés, Dietz, \& Mendoza Zuany, 2016).

\section{Discusión teórica, hacia una ontología de la universidad}

\section{El sentido de la universidad como resistencia}

Tal vez la pregunta por la esencia de la Universidad permita recorrer el camino de ella misma; recorrerlo como lo afirma Heidegger (1994): el camino del pensar, pensar la universidad. ¿Cómo no pensar la Universidad? Diría Derrida, cómo no representarnos su camino o, en palabras de Heidegger, ¿cómo no ir hasta su origen? (1960).

La cuestión planteada por J. Derrida nos permite construir la ontología de la universidad a la vez que recorremos el camino de pensarla. Tener una «razón de ser» es tener una justificación para existir, tener un sentido, una finalidad, una destinación (Derrida, 1997: 1). De esta manera, corresponde nombrar su crisis como su esencia. Una ontología de la universidad se podría concretar en la idea de una universidad en crisis o la permanente crisis de la idea de universidad. El principio de razón da cuenta de la crisis de la universidad (Derrida, 1997).

La universidad moderna, según Heidegger, tiene sus fundamentos en el principio de la razón; pero éste aún no es pensado, interrogado, cuestionado, respecto de su procedencia (Derrida, 1997). Este principio de razón está invisibilizado en la universidad de la tecnociencia; no hay una preocupación por ello, permanece ahí en el ocultamiento, en lo impensado, en la oscuridad que puede ser provechosa para ciertos fines: progreso científico, innovación, competitividad, calidad.

De acuerdo con Derrida, centrar la atención en los problemas latentes y fundantes de la universidad del presente hace que lleguemos a pensar su principio de razón, el cual se instaura al pensar lo fundamental ya sea en cuanto a su razón de ser, a su misión o a las políticas de enseñanza e investigación (1997). Como lo advierte Habermas, el hecho de que las ciencias adopten para los Estados el lugar de primera fuerza productiva, trae consecuencias para el sistema científico e incide sobre el sentido y fines atribuidos a la universidad (Habermas, 2012). 
En este contexto adquiere primacía la tensión entre teoría y práctica; los cuestionamientos a la legitimidad de la universidad se originan en la exigencia de que el conocimiento posea un correlato con la práctica, en gran parte debido a los problemas de desaceleración económica que demandan de la investigación una acción terapéutica financiada por la industria y sometida a un control permanente sobre las universidades (Santos, 2007).

Debates de este tipo se remontan a los primeros siglos de establecimiento de la universidad moderna, como lo menciona el mismo Habermas citando a Fichte y a Schleiermacher (2012). La crisis ontológica de la universidad se devela en el conflicto y la tensión por el poder, manifestado en las confrontaciones externas e internas que ya Kant había mostrado en su texto "El Conflicto de las Facultades"; allí se ilustra cómo el valor de la autonomía, que ha definido ontológicamente a la universidad durante su historia moderna, se enfrenta a las pretensiones de manipulación y control del poder exterior.

Kant analiza que para su época se constituyeron dispositivos externos que terminaron controlando y recortando la libertad del pensar y del saber, invistiendo con cargos públicos a los "doctos" o "letrados", para instrumentalizarlos en función de las pretensiones del gobierno y de sus propios intereses.

Entonces es el gobierno de las naciones la primera fuerza interesada en ejercer poder sobre la universidad. Reducir al profesional o al egresado de un programa universitario a un simple funcionario que cumple con unos requerimientos técnicos propios del cargo público, ha sido un problema encontrado ya por Kant en la universidad alemana del siglo XVIII; éste llama "negociantes o peritos del saber" a los funcionarios que después de haber sido formados en las teorías propias de su carrera universitaria, terminan como instrumentos de la voluntad del dominante, a cambio de un buen salario o de una posición de poder en el gobierno o en las instancias académicas.

Kant señala una doble división funcional de la universidad, determinada por el poder del Estado: las "Facultades Superiores" y la "Facultad Inferior"; las primeras organizadas en orden jerárquico son Medicina, Derecho, Teología, que obran sin autonomía porque se dedican a preparar funcionarios basados en los manuales definidos por el poder externo. No obran inspiradas en la razón, pues si así lo hicieren "[...] vulnera con ello la autoridad que el gobierno ejerce a través suyo e invade el campo de la Facultad de Filosofía [...]" (Kant, 2003: 70).

En la universidad alemana contemporánea de Kant, pensar y juzgar con autonomía, era propiedad de la Facultad de Filosofía porque desde allí se examinaba la verdad de las demás disciplinas, por lo cual "[...] tiene que ser concebida como sujeta tan solo a la legislación de la razón y no a la del gobierno [...]" (Kant, 2003: 76). Entonces, una facultad que tiene atribuciones de pensar 
la verdad termina enfrentada con otras dependencias que aunque en la esfera del obrar tienen fuerza y poder por su posibilidad de incidir en el pueblo, deben someterse al escrutinio de aquella que tiene asegurada para sí el atributo del pensar las verdades de las disciplinas. Ello, como es natural, incomoda a las facultades superiores que ven su doctrina permanentemente cuestionada por la Facultad Inferior, pues como dice el mismo Kant, ésta "[...] tiene el deber de velar porque, si bien no se diga públicamente toda la verdad, si sea verdad todo lo que se diga y sea establecido como principio" (Kant, 2003: 83).

Así, el gobierno, según Kant, mantiene un control de lo que enseñan en las Facultades Superiores, y por ende, de la manera como los profesionales que de allí pasan a ser sus funcionarios influyen en el pueblo, que está más orientado hacia aquello que le implique un menor esfuerzo intelectual; además la Facultad Inferior o de Filosofía no goza de buen aprecio por el gobierno porque no puede controlar su libre examen. En los tres casos, frente al gobierno, frente a las Facultades superiores y frente al pueblo, la Facultad Inferior o de Filosofía es considerada como peligrosa para el statu quo. De esta manera se configura el conflicto en la universidad, el cual se concreta en el campo del poder; la universidad vive su crisis tanto interna como externa alrededor de la lucha por el poder.

\section{Bolonia o la mercantilización de la universidad}

Un estudio que permite analizar la crisis de la universidad, a través de la actualización del conflicto de las facultades de Kant, es el que realiza Pierre Bourdieu (2012) en su obra Homo Academicus, donde concluye que la distribución del capital cultural estratifica a los profesores en dominados y dominadores, en primer lugar, los ubica dentro del grupo de los "dominados" respecto del poder opuestos a los patrones de la industria y el comercio. Pero, en el campo de la producción cultural, se diferencian de los artistas e intelectuales independientes, ubicándose respecto de ellos como clase dominante por adscribirse a una forma institucionalizada de capital cultural (Bourdieu, 2012). Así, el poder de dominación al interior de la universidad se da no sólo entre facultades, sino entre estratificación docente según la facultad a la que estén adscritos y el nivel de institucionalización de la misma.

Según el estudio de Bourdieu, los profesores universitarios más cercanos a los altos funcionarios, tienen mayor reconocimiento, poder, influencia, y respetabilidad; son la clase dominante con poder político y económico. Pero la otra clase de profesores incluye a los que han adquirido prestigio cultural. En definitiva, lo que se quiere mostrar es cómo el conflicto de las facultades en la universidad 
actual se mantiene en la reproducción de campo del poder y se evidencia en la oposición entre la fracción dominada y la fracción dominante (Bourdieu, 2012).

La tensión actual es producto de la reconfiguración del sistema universitario como respuesta a los requerimientos del modelo neoliberal que privilegia la generación de conocimiento útil, en especial aquel generador de capital mediante la innovación tecnológica; por ello aumenta su poder en el terreno del consumo, discrimina y excluye la investigación y la formación en artes y humanidades justificados en el discurso de la sociedad del conocimiento.

De acuerdo con Boaventura de Souza Santos (2007), la idea de la sociedad del conocimiento ha derivado en una concepción de éste como mercancía, en la idea del capitalismo como organizador de las relaciones sociales y en los valores de la competitividad, la privatización, la mercantilización y la globalización, como factores legitimadores de cualquier acción. Esto, según Lyotard (1990), ha cimentado una nueva identidad universitaria en la cual la pregunta por la verdad se transforma en la pregunta por la utilidad.

El acuerdo de Bolonia se instala como referente discursivo fundamentado, según Zygmunt Bauman, en tres categorías: globalización, movilidad y consumo (2001). En la actualidad la palabra "globalización" se usa como antídoto o respuesta canónica para dar sustento o explicar la búsqueda del progreso, la libertad y la felicidad, pero como pensamiento único excluye, señala penuria, devastación y degradación de un mundo sometido a la exigencia de la globalidad que segrega, separa y margina (Bauman, 2001).

La movilidad en el Espacio Europeo de Educación Superior está garantizada por la uniformidad en los curricula y por la flexibilidad para asegurar titulaciones; esta estrategia se piensa para favorecer el modelo universitario dominante en menoscabo de los modelos locales, trayendo como consecuencia el desplazamiento del poder de los Estados, la pérdida de la autonomía y la libertad, y generando miseria en los espacios locales que carecen de la

Con la pérdida de financiación por parte del Estado, las universidades se vieron abocadas a adaptarse a las exigencias de la economía, convirtiéndose en un mercado en sí misma, razón por la cual los estudiantes dejan de acceder en calidad de ciudadanos y empiezan a hacerlo en calidad de consumidores debido a la eliminación de la gratuidad de la educación y a la sustitución de becas de estudio por onerosos préstamos (Santos 2007, Giroux 2008).

El auge de los cursos en línea amplía la mercantilización del conocimiento y convierte un valioso recurso didáctico en una tecnología que se transforma en eficiencia estandarizada, lo cual, según Marcuse, confina la libertad "[...] a la selección del medio más adecuado para alcanzar una meta, la cual el individuo no fijó" (Citado por Giroux, 2008: 152). Henry Giroux (2008: 24) va más allá al 
indicar que el cinismo en la política y el escepticismo frente a la educación han lesionado el compromiso de defender las instituciones que son vitales y cruciales para una democracia, y han devenido en una aceptación de que la educación ha de contribuir a la fuerza laboral y a la filosofía de seguridad nacional en Estados Unidos.

La subordinación de investigaciones médicas y biotecnológicas a las grandes multinacionales son evidencia clara de la pérdida de independencia de las universidades, aspecto nodal de su esencia democrática e indispensable para formar en pos del bien público. De igual forma, el auge del militarismo, encarnado en la seguridad como imperativo del Estado, utiliza el conocimiento como dispositivo de control de todas las esferas de la vida, no sólo en los campos de la ingeniería, las telecomunicaciones y en general las ciencias naturales, sino también en las ciencias sociales y en enfoques tradicionalmente contradictores como la teoría crítica y el análisis del discurso (Giroux, 2008).

Giroux propone que la universidad cuestione e interrogue el papel de los intelectuales acerca de su responsabilidad social, pedagógica y política para enfrentar la militarización y la mercantilización del conocimiento, y sugiere que los docentes mantengan la convicción de que el aprendizaje está al servicio del bien público, algo que debe cimentarse en la duda para promover la democracia y tener como objetivo un futuro que amplifique la libertad humana y promueva la justicia social (2008).

En el mismo sentido, Martha Nussbaum defiende la formación humanística en el contexto de las universidades, para que los estudiantes accedan a una comprensión de la diversidad social y sean activos partícipes de una sociedad democrática. No obstante, muchas universidades se orientan más a una formación tecnocientífica que satisface el mercado, por lo cual se presenta un recorte de recursos para los programas humanísticos con las consecuentes debilidades pedagógicas en su implementación (2010).

Las humanidades enfrentan a las demandas de los gobiernos, en especial en Gran Bretaña, donde compiten por financiación para las investigaciones a partir del criterio de impacto y teniendo que adaptarse a las lógicas productivas del mercado, con lo cual renuncian a la valoración de la investigación humanística en términos de reconocimiento de las formas diversas de encontrarnos con la condición humana. Ello genera un desplazamiento de las universidades locales debido a la presión de las dominantes, como bien lo ejemplifica Nussbaum al citar el caso de la universidad de Glasgow en Escocia:

[...] El discurso inaugural de la universidad de Saint Andrews pronunciado por John Stuart Mill, alaba la aptitud del sistema universitario es- 
cocés para la ciudadanía democrática en comparación con los contenidos de la formación universitaria en Inglaterra, más restringidos a la teología. Sin embargo, la normalización del sistema de educación superior en la Unión Europea, impuesta por el proceso de Bolonia, ha obligado a Escocia a asimilarse al resto de Europa, en lugar de hacer lo contrario (Nussbaum, 2010: 171).

\section{La universidad en Latinoamérica: decolonialidad y liberación}

Pensar la ontología de la universidad en Latinoamérica exige de una filosofía que oriente su razón de ser. La Filosofía de la Liberación traza los horizontes de pensamiento para pensar una universidad latinoamericana. Enrique Dussel señala la configuración de su pensamiento a partir de la contrastación entre su experiencia filosófica europea y el conocimiento de la lucha de los excluidos, que le permitió ver "el mundo desde abajo", que condujo a criticar las bases filosóficas de corte europeo. Asimismo enfatiza la ruptura con el mito de la Grecia aristocrática que soporta el posterior sentido humanístico de la modernidad, la crítica al desarraigo individualista y burgués del Emilio de Rousseau y el cuestionamiento a la integración del conocimiento técnico universal con las singularidades de cada contexto, propuesto por Ricoeur, por no considerarlo apto para el encuentro asimétrico entre dos culturas (Dussel, 1998).

De igual forma, plantea sus diferencias frente a cada una de las generaciones de la filosofía crítica. Si bien a la primera le acepta el reconocimiento de la materialidad expresada en el sufrimiento, la pobreza y el hambre, cuestiona que se centren en el obrero dejando de lado a los despojados de la periferia, una exclusión material propia de la dominación colonizadora (Dussel, 2015). Con respecto a la segunda generación, reconoce la superación del paradigma solipsista de la conciencia propio de la primera, y las implicaciones éticas y políticas de la búsqueda del encuentro con el otro a través de las comunidades simétricas de diálogo; pero cuestiona su desconocimiento de las condiciones materiales de los participantes y su consensualismo idealista que silencia la voz de los excluidos. Sustenta, por lo tanto, que esas comunidades intersubjetivas reconozcan la corporalidad viviente del deliberante, pues discusiones ajenas al hambre, al desempleo, al despojo del cuerpo y la marginalidad de la mujer, podrán ser válidas en países del centro, pero en países periféricos son inaceptables (Dussel, 2015). 
Por lo tanto, propone la construcción de una comunidad de diálogo de los excluidos que confronte a la anterior de corte hegemónico. Opera así una reconstrucción de los grupos sociales que, en contravía de las sociedades políticas desmaterializadas propuestas por Arendt, efectúan reivindicaciones materiales (corporales, ambientales) para constituirse en sociedades políticas, que luchan por el reconocimiento de la diferencia, inscrita en condiciones de exclusión no reconocidas por el poder hegemónico (Dussel, 2015).

La filosofía de la liberación crea así una exigencia reflexiva al sentido de la universidad en América Latina. La esencia de la misma debe basarse en una visión crítica del conocimiento que parta de la reflexión acerca de las condiciones de legitimidad de la universidad en la sociedad. A partir de la década de 1960 opera una doble crítica a la universidad; la primera cuestiona que la universidad no haya volcado su conocimiento acumulado al servicio de la solución de los problemas sociales, y la segunda la juzga por no poner su autonomía y su espíritu crítico al servicio de los grupos sociales (Santos B. D., 1998).

La universidad se fractura en su identidad cultural y social debido a la tensión entre la demanda hiperprivada y la hiperpública. Ello demanda un resistencia contrahegemónica que supere la naturalización del proyecto de la globalización neoliberal y proponga un concepto de globalización alternativa, pero para ello debe articular la capacidad de autoregenerarse con la de concitar la injerencia de otros actores y fuerzas sociales que cohabitan en ella. Esta aspiración encuentra en los sectores sociales colectivamente organizados (sindicatos, asociaciones, organizaciones no gubernamentales, comunidades indígenas) una posibilidad de integración, a pesar de que estos sectores han percibido a la universidad como elitista.

Una forma de luchar por la legitimidad ante la pérdida de su hegemonía en relación con el conocimiento, Santos propone el fortalecimiento de becas en vez de créditos bajo el contrapago de trabajo social universitario, garantías de acceso y acompañamiento con una posición afirmativa de inclusión racial y social que supere la visión elitista de la Meritocracia. Pero estas acciones demandan una concepción diferente del conocimiento y la investigación, por lo que la propuesta de un diálogo de saberes se torna indispensable, así como la implementación de un saber práctico, que en consonancia con la comunidad material de Dussel (2015) supere la actitud funcionalista de la investigación y mediante la investigación-acción armonice los intereses científicos de la universidad, con las necesidades concretas de los grupos sociales que participen de ésta (Santos B. D., 2007).

Lo anterior debe desarrollarse a partir de una ecología de saberes que elimine la injusticia cognitiva, para superar los desplazamientos que el conocimiento hegemónico hizo de los saberes populares y tradicionales, y la desarticulación so- 
cial y cultural generada por las visiones economicistas del saber tecnocientífico. Es un intento de vinculación y diálogo entre esos saberes culturales que circulan socialmente y los saberes científicos que produce la universidad.

De esta forma la universidad encontrará su razón de ser en la constitución de una crítica de la razón política (Dussell, 1999), que busque su ontología en el encuentro con el otro:

El que actúa bajo la responsabilidad por el Otro y en cumplimiento de las indicadas exigencias, puede tener la pretensión de establecer un orden más justo. Si efectivamente a mediano o a largo plazo el dicho orden fuera efectivamente más justo, lo juzgará la historia (Dussell, 1999: 195).

\section{A modo de cierre}

La deslegitimación de la universidad humanista por parte de la tecnociencia y de la sociedad del conocimiento son los elementos constituyentes del trayecto ontológico; de constitución del sentido y de la identidad de la universidad:

[...] las universidades y las instituciones de enseñanza superior son de ahora en adelante solicitadas para que fuercen sus competencias, y no sus ideas [...] La transmisión de los saberes ya no aparece como destinada a formar una élite capaz de guiar a la nación en su emancipación [...] (Lyotard, 1990: 90).

En el ejercicio de revisión teórica se observó que cada momento de crisis de la universidad en la sociedad forma parte de su esencia. Entonces diríamos con Heidegger, que la pregunta por la esencia de la universidad es la pregunta por su sentido, por su camino. En todos los casos se encuentra que la universidad está en crisis permanente y es la crisis la que la constituye y reconstituye. Esta crisis le es connatural, es su ontología.

Crisis de legitimidad porque por andar anclada en la búsqueda de la verdad no fue útil al mercado; porque no respondió a los requerimientos de la sociedad del conocimiento; es el funcionalismo cuestionando y solicitando la reforma de la universidad para que atienda las demandas del capital, porque cuando se transformó en institución funcional que respondió al capitalismo, excluyó los principios de humanización; formación política, justicia, democracia, arte, con enfoque planetario sostenible.

Pero, en definitiva, crisis que da lugar a la resistencia y a la esperanza en una universidad para la liberación. Una universidad alternativa que asuma el reto de 
construir, interpretar o develar verdades tanto de la ciencia como del contexto ancestral; que aporte al desarrollo tecno-científico y económico en condiciones de igualdad, dentro de una sociedad plural, diversa y justa con los seres humanos que la integran y con el espacio que habitan.

\section{Referencias}

Ahedo-Ruiz, Joshu (2016). La universidad una escuela al servicio de la verdad. Revista Complutense de Educación, 12 (2), 517-532.

Alba-Pastor, Carmen (2005). El profesorado y las tecnologías de la información y la comunicación en el proceso de convergencia al Espacio Europeo de Educación Superior. Revista de Educación, 337, 13-36.

Alba-Pastor, Carmen y Carballo-Santaolalla, Rafael (2005). Viabilidades de las propuestas metodológicas para la aplicación del crédito europeo por parte del profesorado de las universidades españolas, vinculadas a la utilización de las TIC en la docencia y la investigación. Revista de Educación, 337, 71-97.

Ávila-Romero, León; Betancourt-Posada; Alberto, Arias-Hernández, Gabriela \& Ávila-Romero, Agustín (2016). Vinculación comunitaria y diálogo de saberes en la educación superior intercultural en México. Revista Mexicana de Investigación Educativa, 21 (70), 759-783.

Bartual-Figueras, María Teresa \& Turmo-Garuz, Joaquín (2016). Educación superior y competencias para el empleo. El punto de vista de los empresarios. Revista Complutense de Educación, 27 (3), 1211-1228.

Bauman, Zygmunt (2001). La globalización. Consecuencias humanas. Mexico: Fondo de Cultura Económica.

Benítez, Cristina (1996). Desafíos de la universidad contemporánea. Los casos de Alemania, Estados Unidos y América Latina. Revista Mexicana de Investigación Educativa, 1 (1), 249-253.

Bombillar-Sáenz, Francisco (2010). Bolonia y Pisa: algo más que dos ciudades italianas. Una reflexión acerca de los nuevos retos a los que se enfrenta la educación universitaria en España. Zona Próxima, 12, 208-221.

Bourdieu, Pierre (2012). Homo academicus. (A. Dilon, Trad.). Buenos Aires: Siglo xxI editores.

Cabello-Martínez, Josefa \& Antón-Ares, Paloma (2005). Conversaciones con el profesorado. Un estudio en cuatro universidades españolas sobre el Espacio Europeo y el uso de las Tic. Revista de Educación, 337, 149-167.

Calvo- Bernardino, Antonio \& Mingorance- Arnaiz, Ana (2009). La estrategia de las universidades frente al Espacio Europeo de Educación Superior. Revista Complutense de Educación, 20 (2), 319-342.

Canales, Alejandro (2011). El dilema de la investigación universitaria. Perfiles Educativos, 33, 34-44.

Castillo Rosas, Adriana (2016). Reconstrucción histórico-política de la educación indígena en México y los antecedentes no oficiales de la Universidad Inter- 
cultural del Estado de Hidalgo. Revista Mexicana de Investigación Educativa, 21, (70) , 691-717.

Castro-Estrada, María Luisa; Rodríguez-Rejas, María José \& Urteaga-Urías, Emiliano (2016). Abrir las aulas: el vínculo entre docencia, investigación y vinculación comunitaria. Revista Brasileira de Educao. 21 (66), 737-758.

Cuño Bonito, Justo (2016). La universidad Latinoamericana en la encrucijada: amenazas, desafíos y soluciones. Revista Historia de Educación Latinoamericana, 18 (26), 241-277.

De Pons, Juan Pablo \& Villaciervos-Moreno, Patricia (2005). El Espacio Europeo de Educación Superior y las tecnologías de la información y la comunicación. Percepciones y demandas del profesorado. Revista de Educación, 337, 99-124.

De Vries, Wietse e Ibarra-Colado, Eduardo (2004). La gestión en la universidad: interrogantes y problemas en busca de respuestas. Revista Mexicana de Investigación educativa, 9 (22), 575-584.

Derrida, Jacques (1997). Las pupilas de la Universidad. El principio de razón y la idea de la Universidad. Barcelona: Proyecto A Edición digital de Derridá en castellano.

Dussel, Enrique (1998). En búsqueda del sentido (origen y sentido de una filosofía de la Liberación). Anthropos, 180, 74-90.

Dussel, Enrique (2015). Filosofías del sur. México: Akal.

Dussel, Enrique (1999). Seis tesis para una crítica de la razón política (el ciudadano como agente político). Signos Filosóficos. 1 (2) , 171-197.

Egido-Álvarez, Inmaculada, Fernández Díaz, José \& Galán, Arturo (2014). La dimensión social del proceso de Bolonia: apoyos y servicios para grupos de estudiantes poco representados en las universidades españolas. Educación xx1, $17(2), 57-81$.

García-Nieto, Narciso, Asensio-Muñoz, Inmaculada, Carballo-Santaolalla, Rafael; García- García, Mercedes \& Guardia- González, Soledad (2005). La tutoría universitaria ante el proceso de armonización europea. Revista de Educación, 337, 189-210.

García-Peñalvo, Francisco (2016). La tercera Misión. Teoría de la Educación, 17 (1), 7-18.

Gargallo-Bernardo; Suárez, Jesús; Garfella, Pedro \& Fernández, Amparo (2011). El cuestionario CEMEDEPU. Un instrumento para la evaluación de la metodología docente y evaluativa de los profesores universitarios, Estudios Sobre Educación, 21, 9-40.

Geneyro, Juan Carlos (1997). Educación superior: Democracia y Globalización. Seminario Internacional: Las nuevas universidades del final del siglo xx (págs. 242-262). Buenos Aires: Universidad General Sarmiento.

Gijón-Puerta, José \& Crisol Moya, Emilio (2012). La internacionalización de la Educación Superior. El caso del Espacio Europeo de Educación Superior, REDU: Revista Docencia Universitaria, 10 (1), 389-414.

Giroux, Henry (2008). La Universidad Secuestrada. El reto de enfrentar a la alianza militar-industria-académica. (E. Pereira Urosevic, Trad.) Caracas: Centro Internacional Miranda.

Habermas, Jürgen (2012). Teoría y praxis. Estudio de filosofía social. Madrid. 
Heidegger, M. (1960). Sendas perdidas. (J. Rovira Armencol, Trad.) Buenos Aires: Lozada S. A.

Heidegger, Martin (1994). Conferencias y artículos. (E. Barjau, Trad.) Barcelona: Ediciones del Serbal.

Hoyos, Guillermo (2008). Educación para un nuevo humanismo en tiempos de globalización. Encuentro de profesores que enseñan filosofía. Tunja: UPTC, 1-2

Huber, Günter (2008). Aprendizaje activo y metodologías educativas, Revista de Educación (Número Extraordinario), mayo-agosto, 59-81.

Ibarra Colado, Eduardo (2002). Capitalismo académico y universidad. La universidad reinventada. Revista de la Educación Superior, 122, 145-152.

Ibarra Colado, Eduardo (2005). Origen de la empresarizalización de la universidad: el pasado de la gestión de los negocios en el presente del manejo de la universidad. Revista de la Educación Superior, 34(134), 13-37.

Ibarra, Noelia \& Ballester, Joseph (2016). La literatura en la formación universitaria desde el Espacio Europeo de Educación Superior. Alpha; revista de artes, letras y filosofía (Chile) 43, 303-317.

Ion, Gerogeta \& Cano, Elena (2012). La formación del profesorado universitario para la implementación de la evaluación por competencias. Educación xx1, 15 (2), 249-270.

Jiménez-Ramírez, Magdalena (2017). Los nuevos estudios de doctorado en España: Avances y retos para la universidad. Revista Iberoamericana de Educación Superior, 8 (21), 123-137.

Kant, Immanuel (2003). El conflicto de las facultades. (R. Aramayo, Trad.) Madrid. Alianza Editorial.

Lampert, Ernani (2009). Recrear la universidad: una premisa urgente. Perfiles Educativos, 126, 100-114.

Llamas-Tascón, Angel (1997). Universidad y Nuevos Temas a fin de siglo. Seminario Internacional: las nuevas universidades del final del siglo xx. San Miguel, Argentina: Universidad Nacional del General Sarmiento.

Lozada-Barahona, Nelson, Gutiérrez-Vargas, Liliana (2014). La naturaleza única y particular de la universidad latinoamericana en la construcción de la sociedad. Revista Internacional de Sociología, 72 (1), 203-248.

Lyotard, Jean Francois (1990). La condición posmoderna. Informe sobre el saber. Buenos Aires: Teorema.

Malagón Plata, L. (2003). La pertinencia en la educación superior: elementos para su comprensión. Revista de la Educación Superior, 32 (127), 113-134.

Mollis, M. (2014). Administrar la crisis de la educación pública y evaluar la calidad universitaria en América Latina: dos caras de la misma reforma educativa. Revista de la Educación Superior, 33 (169), 25-45.

Margaleff-García, Leonor, Álvarez-Méndez, Juan Manuel (2005). La formación del profesor universitario para la innovación en el marco de la integración del espacio europeo de la educación superior. Revista de Educación, 337, 51-70.

Mas-Torelló, Oscar \& Olmos-Rueda, Patricia (2016). El profesor universitario en el Espacio Europeo de Educación Superior. La auto percepción de sus competencias docentes actuales y orientaciones para su formación pedagógica. Revista Mexicana de Investigación Educativa, 21 (69), 439 - 470. 
Mateo, Joan \& Vlachopoulos, Dimitrios (2013). Reflexiones en torno al aprendizaje y a la evaluación en la universidad en el contexto de un nuevo paradigma para la educación superior, Educación xx1, 16(2), 183-208.

Mateos-Cortés, Laura; Dietz, Gunther \& Mendoza Zuany, Guadalupe (2016). ¿Saberes haceres interculturales? Experiencias profesionales y comunitarias de egresados de la educación superior intercultural veracruzana. Revista Mexicana de Investigación Educativa, 21 (70), 809-835.

Mateos-Cortés, L. S., \& Dietz, Gunther (2016). Universidades interculturales en México. Balance de la primera década. Revista Mexicana de Investigación Educativa, 21 (70) , 683-690.

Michavila, Francisco (2005). No sin los profesores. Revista de Educación, 337, 3749.

Montero-Curiel, Marisa (2010). El Proceso de Bolonia y las nuevas competencias, Tejuelo, 9 (1), 19-37.

Muñoz-Sedano, Antonio (1998). Enfoques y modelos de educación Multicultural e Intercultural, Revista Complutense de Educación, 9 (2), 101-135.

Nussbaum, Martha (2010). Sin fines de lucro. ¿Por qué la democracia necesita de las humanidades? (M. V. Rodil, Trad.) Buenos Aires: Katz Editores.

Olivera-Beltrán, Javier (2000). El Informe Universidad 2000 y los Centros Superiores de Ciencias de la Actividad Física y el Deporte, Apuntes Educación Física y Deporte, 60 (2), 1-4.

Rodríguez-Gómez, Roberto (1997). Las universidades modernas, Revista Mexicana de Investigación Educativa, 2 (4), 365-368.

Rodríguez-Gómez, Roberto (2001). La universidad latinoamericana y el siglo XX: algunos retos estructurales, en C. L. Sociales, Paulo Freire y la agenda de la educación latinoamericana en el siglo XXI (págs. 53-80). Buenos Aires: Clacso.

Runge-Peña, Andrés K. (2005). Reflexiones pedagógicas sobre la investigación y la docencia universitarias a la luz de la formación: el modelo humboldtiano como ejemplo. Unipluridiversidad ,5 (2). Descargado de: https:/ / aprendeenlinea.udea.edu.co/revistas/index.php/unip/article/view/12151/11032, (consultado.

Salcines, José. V.; Freire Seoane, María \& Orosa González, Javier (2008). La universidad española en el siglo xxi. Como afrontar los retos de la globalización. Revista de la Educación Superior, 147, 45-61.

Santos, Boaventura De Souza (1998). De La Mano de Alicia: Lo social y lo político en la Postmodernidad, Bogotá: Ediciones Uniandes.

Santos, Boaventura De Souza (2007). La Universidad en el siglo XXI, para una reforma democrática y emancipatoria de la universidad. La Paz: CIDES-UMSA.

Santos, Boaventura De Souza (2010). La Universidad Europea en la Encrucijada, El Viejo Topo, 274, 48-55.

Schmelkes, Silvia (2008). Las universidades interculturales en México: ¿una contribución a la equidad en educación superior?. Disponible en http://www. mineducacion.gov.co/cvn/1665/articles-175893_archivo_pdf2.pdf. (

Soto-Arango, Diana y Romero-Forero, Aracely (2016). La Universidad Latinoamericana y del Caribe en los desafíos del Siglo xxI. Historia de la educación Latinoamericana, 18 (26), 279-309. 
Torres, Carlos Alberto (1997). La universidad latinoamericana, de la reforma de 1918, al cambio estructural de los noventa, Revista Mexicana de Investigación Educativa, 4 (8), 345-377.

Torres, Carlos Alberto (2014). Las universidades públicas y el sentido común neoliberal: siete tesis iconoclastas, Revista Latinoamericana de Políticas y Administración de la Educación ,1 (1 ) 1, 18-31.

Trujillo, Juan Manuel; Hinojo Lucena, Francisco \& Aznar-Díaz, Inmaculada (2011). Propuestas de trabajo innovadoras y colaborativas e-learning 2.0 como demanda de la sociedad del conocimiento. Estudios sobre educación, 20, 141-159.

Vega-Cantor, Renán (2015). La universidad de la ignorancia. Capitalismo académico y mercantilización de la educación superior. Bogotá: Ocean Sur.

Williamson, Guillermo (2004). ¿Educación multicultural, educación intercultural Bilingüe, educación indígena o educación intercultural? Cuadernos interculturales, 02 (3), 23-34. 